\title{
REGULARITY OF BAIRE MEASURES
}

\author{
N. DINCULEANU AND PAUL W. LEWIS ${ }^{1}$
}

Abstract. In a recent paper N. Dinculeanu and I. Kluvánek showed that any Baire measure with values in a locally convex topological vector space is regular. Their construction depended heavily on the regularity of nonnegative Baire measures. In the present paper, a proof of the regularity is given which holds at once for the nonnegative case and the vector case.

I. The literature contains an abundance of proofs of the regularity of Baire measures. However, many of them, such as the one found in [4], require the proofs of a considerable number of preliminary lemmas before the actual theorem can be derived.

On the other hand, the proof of the regularity of vector-valued Baire measures found in [1] uses the regularity of positive Baire measures.

The following approach seems not only to require a minimum of machinery, but it also applies directly to the vector-valued measures considered in [1].

II. Let $T$ denote a locally compact Hausdorff space, $\Delta$ the Baire $\sigma$-ring of subsets of $T, X$ a locally convex topological vector space whose topology is given by a family $\left\{|\cdot|_{p}\right\}_{p \in P}$ of seminorms, and $m: \Delta \rightarrow X$ a (countably additive) Baire measure on $\mathrm{T}$.

We say that $m$ is regular at a set $A \in \Delta$ provided that for each neighborhood $V$ of $0 \in X$ there is a compact set $K \in \Delta$ and an open set $G \in \Delta$, with $K \subset A \subset G$, so that $m(S) \in V$, for each $S \in \Delta, S \subset G-K$. Such a pair $(K, G)$ is said to be appropriate for $(A, V)$. The measure $m$ is said to be regular provided it is regular at each $A \in \Delta$.

For $p \in P$, we consider the $p$-quasi-variation $\tilde{m}_{p}$ defined for every $A \in \Delta$ by $\tilde{m}_{p}(A)=\sup \left\{|m(S)|_{p}: S \subset A, S \in \Delta\right\}$.

LEMma. The p-quasi-variation is positive, increasing, countably subadditive, and $\tilde{m}_{p}(A)<\infty$ for each $A \in \Delta$. Furthermore, $A_{n} \searrow \varnothing$ implies that $\tilde{m}_{p}\left(A_{n}\right) \searrow 0$, i.e. $m$ is vsr (see [2, III, 4.5] and [3, Theorems 2.6 and 2.7]).

Received by the editors November 5, 1969.

AMS 1969 subject classifications. Primary 2850; Secondary 2635.

Key words and phrases. Baire $\sigma$-ring, regular Baire measures, p-quasi-variation, monotone ring of sets.

${ }^{1}$ A portion of this research was done while the second author was at the University of Utah; he is now at North Texas State University. 
We are now able to prove the following theorem.

TheOREM. Every Baire measure $m: \Delta \rightarrow X$ is regular.

Proof. Let $\mathrm{e}$ denote the class of sets of $\Delta$ for which $m$ is regular. We shall prove that $\mathfrak{C}$ coincides with $\Delta$.

(a) $\mathcal{e}$ contains the compact $G_{\delta}$ sets. In fact, let $K$ be a compact $G_{\delta}$ and let $\left\{G_{n}\right\}$ be a decreasing sequence of open Baire sets so that $G_{n} \searrow K$. Let $p \in P, \epsilon>0$, and $V=V(p, \epsilon)$ be the open $p-\epsilon$-ball around $0 \in X$. Then $\tilde{m}_{p}\left(G_{n}-K\right) \searrow 0$, and there is an $N$ so that $\tilde{m}_{p}\left(G_{N}-K\right)<\epsilon$. Hence if $S \in \Delta$ and $S$ is contained in $G_{N}-K$, then $\tilde{m}_{p}(S)<\epsilon$. Thus $m(S) \in V$, and it follows that $m$ is regular at $K$.

(b) $\mathcal{C}$ is a ring. Let $A_{1}, A_{2} \in \mathcal{C}$, and suppose that $V=V(p, \epsilon)$ is a neighborhood of $0 \in X$. Let $\left(K_{i}, G_{i}\right)$ be appropriate for $\left(A_{i}, V / 2\right)$, $i=1,2$. Then $\left(K_{1} \cup K_{2}, G_{1} \cup G_{2}\right)$ is appropriate for $\left(A_{1} \cup A_{2}, V\right)$. For if $S \in \Delta$ and $S$ is a subset of $\left(G_{1} \cup G_{2}\right)-\left(K_{1} \cup K_{2}\right)$, then $S$ can be written as $S_{1} \cup S_{2}, S_{i} \in \Delta, S_{1} \subset G_{1}-K_{1}$, and $S_{2} \subset G_{2}-K_{2}$. Therefore $m\left(S_{1}\right)$ $+m\left(S_{2}\right) \in V$, and $A_{1} \cup A_{2} \in \mathcal{e}$.

In addition, it follows that $\left(K_{1}-G_{2}, G_{1}-K_{2}\right)$ is appropriate for $A_{1}-A_{2}$. For

$$
\left(G_{1}-K_{2}\right)-\left(K_{1}-G_{2}\right)=\left[G_{1}-\left(K_{1} \cup K_{2}\right)\right] \cup\left[\left(G_{1} \cap G_{2}\right)-K_{2}\right] .
$$

Therefore, if $S \in \Delta$ and $S \subset\left(G_{1}-K_{2}\right)-\left(K_{1}-G_{2}\right)$, then $S=S_{1} \cup S_{2}$, where $S_{1} \subset G_{1}-K_{1}$ and $S_{2} \subset G_{2}-K_{2}$. Thus $m\left(S_{1}\right)+m\left(S_{2}\right) \in V$, and it follows that $\mathfrak{C}$ is a ring.

(c) $\mathbb{C}$ is a monotone class of sets. In fact, suppose that $\left\{A_{n}\right\}$ is a decreasing sequence of sets in $\mathrm{C}$, and let $A=\cap A_{n}$. Let $V=V(p, \epsilon)$ be a neighborhood of $0 \in X$ as above, and for each $n$ choose an appropriate pair for $\left(A_{n}, V / 2^{2(n+1))}\right.$. By taking intersections, we can generate a sequence $\left\{\left(K_{\dot{n}}, G_{n}\right)\right\}$ so that, for each $n,\left(K_{n}, G_{n}\right)$ is appropriate for $\left(A_{n}, V / 2\right)$, and each of the sequences $\left\{K_{n}\right\}$ and $\left\{G_{n}\right\}$ is decreasing. The set $K=\cap K_{n}$ is compact, $K$ belongs to $\Delta$, and there is a positive integer $N$ so that if $n \geqq N$, then $\tilde{m}_{p}\left(K_{n}-K\right)<\epsilon / 2$. Now if $S$ is a subset of $G_{N}-K$, then $S=S_{1} \cup S_{2}, S_{i} \in \Delta, S_{1} \subset G_{N}-K_{N}$, and $S_{2} \subset K_{N}-K$. Therefore $m(S)=m\left(S_{1}\right)+m\left(S_{2}\right) \in V$, and it follows that $A \in \mathbb{C}$.

By a dual argument, one can see that increasing sequences of elements of $\mathfrak{C}$ also belong to $\mathfrak{C}$. Hence $\mathcal{C}$ is a monotone ring containing all the compact $G_{\delta}$ sets, and therefore $\mathcal{C}=\Delta$ (see $[4$, Chapter 1$\left.]\right)$.

REMARK. As is proved in [1], every Baire measure $m: \Delta \rightarrow X$ can be uniquely extended to a regular Borel measure with values in the completion of $X$ (see [1] for the definition of Baire and Borel sets).

REMARK. In order to simultaneously include the case of unbounded positive Baire measures (hence the values would not lie in a locally 
convex space), one may consider the following slightly more general setting. Let $\Delta_{0}$ be the smallest ring and $\Delta_{1}$ the smallest $\delta$-ring generated by the compact $G_{\delta}$ subsets of $T$, let $X$ be a locally convex space, and let $m: \Delta_{1} \rightarrow X$ be a countably additive set function. As before, let $\mathcal{C}$ denote the class of all elements of $\Delta_{1}$ at which $m$ is regular. By the argument above, it follows that $\mathcal{C}$ is a ring containing the compact $G_{\delta}$ subsets of $T$ and the intersection of a decreasing sequence of elements of $\mathcal{C}$ also belongs to $\mathcal{C}$. Furthermore, if $\left\{A_{n}\right\}$ is an increasing sequence of sets in $\mathcal{C}$ so that there is an $A \in \Delta_{1}$ so that $\left\{A_{n}\right\}_{n=1}^{\infty} \subset A$, then $\mathrm{U}_{n=1}^{\infty} A_{n} \in \mathrm{C}$. Thus $\mathrm{C}$ is monotone with respect to $\Delta_{1}$, and it follows that $\Delta_{1}=\mathrm{e}$. To conclude, simply note that $\Delta$ is the collection of all countable unions from $\Delta_{1}$.

\section{REFERENCES}

1. N. Dinculeanu and I. Kluvánek, On vector measures, Proc. London Math. Soc. (3) 17 (1967), 505-512. MR 35 \#5571.

2. N. Dunford and J. Schwartz, Linear operators. I: General theory, Pure and Appl. Math., vol. 7, Interscience, New York, 1958. MR 22 \#8302.

3. G. G. Gould, Integration over vector-valued measures, Proc. London Math. Soc. (3) 15 (1965), 193-225. MR 30 \#4894.

4. P. R. Halmos, Measure theory, Van Nostrand, New York, 1950. MR 11, 504.

5. P. W. Lewis, Some regularity conditions on vector measures with finite semi-variation, Rev. Roumaine Math. (to appear).

Institute of Mathematics, Bucharest, Romania

North Texas State University, Denton, Texas 76201 